\title{
Efecto de la aplicación de solución nutritiva para el crecimiento inicial de Polylepis racemosa a nivel de vivero
}

\section{(Effect of the application of nutritive solution for the initial growth of Polylepis racemosa at hatchery level)}

\author{
Sonia Rosero ${ }^{1}$, José Arcos ${ }^{1}$, Miguel Guallpa ${ }^{1}$, Holguer Guaraca ${ }^{1}$
}

\begin{abstract}
Resumen:
Los programas de forestación y reforestación con énfasis en la sostenibilidad ecológica orientan sus esfuerzos a la protección de fuentes hídricas, la recuperación de áreas con suelos erosionados y el establecimiento de sistemas agroforestales en zonas de altitud elevada donde se requieren plantas de calidad, motivo por el cual se propuso este estudio, para determinar en yagual (Polylepis racemosa), la respuesta en el crecimiento aéreo y foliar en vivero a base de la aplicación de distintas soluciones nutritivas luego del trasplante. El diseño experimental utilizado fue de bloques completamente al azar (BCA) con siete tratamientos y cuatro repeticiones. Los tratamientos consistieron en el uso de una mezcla de tres fertilizantes (nitrato de amonio, nitrato de potasio y fosfato mono potásico) con tres concentraciones (100-50100 (D1); 150-75- 150(D2); 200-100-200(D3) mg.L-1 de NPK respectivamente) y dos frecuencias de aplicación (dos (F1) y tres (F2) veces por semana), más un tratamiento control. Las variables analizadas fueron la altura total (hT) y el número de hojas $\left(\mathrm{N}^{\circ} \mathrm{H}\right)$. Según los resultados, los mejores crecimientos en altura de planta y el mayor número de hojas se obtienen al fertilizar con 200-100-200 mg.L-1 de NPK, con frecuencia de aplicación de tres veces por semana a los 120 días; por el contrario, el menor crecimiento en altura de planta, fue con la combinación D1-F1 y el menor número de hojas reportó un comportamiento similar en los tratamientos D1-F1 y el testigo.
\end{abstract}

Palabras clave: solución nutritiva; crecimiento, fertirriego; Polylepis racemosa.

\begin{abstract}
:
Afforestation and reforestation programs with emphasis on ecological sustainability focus their efforts on the protection of water sources, eroded soils recovery, and the establishment of agroforestry systems in high altitude areas, where quality plants are required. The present study aims to determine the response in aerial and foliar growth of nursery yagual (Polylepis racemosa) to the application of different nutritive solutions after transplantation. A completely randomized blocks (RCB) experimental design was established with seven treatments and four repetitions. The treatments consisted in the use of a mixture of three fertilizers (ammonium nitrate, potassium nitrate and mono potassium phosphate) at three different concentrations (100-50-100 (D1), 150-75-150 (D2), 200-100-200 (D3) mg.L-1 NPK respectively), and two application frequencies (two (F1) and three (F2) times per week), plus a control treatment. The analyzed variables were the total height $(\mathrm{hT})$ and the number of leaves $\left(\mathrm{N}^{\circ} \mathrm{H}\right)$. According to the results, the largest height and the greatest number of leaves were obtained when fertilizing with 200-100-200 mg. L-1 NPK, with three times per week applications for 120 days. On the other hand, the treatment that rendered the shortest plants and lowest number of leaves was the D1-F1 combination, which, in the case of leaf number, was not significantly different from the control.
\end{abstract}

Keywords: nutritious solution; growth, fertigation; Polylepis racemosa.

\footnotetext{
${ }^{1}$ Escuela Superior Politécnica de Chimborazo, Riobamba, Ecuador ( \{s_rosero, jarcos, miguel.guallpa, h.guaraca\} @espoch.edu.ec)
} 


\section{Introducción}

En la zona Andina ecuatoriana, la información disponible sobre fertilización de yagual es escasa o limitada, clasificada como información reservada de ciertos viveros privados tecnificados que son asesorados por especialistas, que participan en el desarrollo y monitoreo de programas de manejo que incluyen la preparación de sustratos, fertirriegos, control de plagas, entre otros. Existe sin embargo, una gran diferencia con el pequeño y mediano viverista, quienes deben producir sus plántulas contando para ello con medios más escasos y tecnologías no tan avanzadas en el sector rural.

En este sentido, la presente investigación busca apoyar técnicamente a estos pequeños y medianos empresarios y orientarlos a mejorar la calidad de su producción. Dada la importancia que tiene la especie Polylepis racemosa al proporcionar bienes y servicios que benefician a los pobladores, cumplen además, con funciones ecológicas, dentro de bosques naturales, plantaciones forestales o sistemas agroforestales (Añazco, 2008). Esta especie tiene gran potencial para la reforestación, la protección de fuentes hídricas y la asociación con cultivos agrícolas en zonas de altura por la alta resistencia a los fríos intensos (Villarpando, Villarpando \& Villalobos, 2008). Razones que sustentan esta investigación a fin de mejorar su etapa de crecimiento inicial en fase de vivero.

La fertilización en yagual es una técnica cultural empleada que pretende encontrar un equilibrio entre los diferentes nutrientes esenciales que se aplican, para que las plántulas formen sus órganos, produzcan los compuestos químicos necesarios para su apropiado funcionamiento, ejecuten sus procesos fisiológicos y reacciones bioquímicas para su desarrollo óptimo (Tisdale et al., 1993).

Existe un amplio consenso en que es necesario proporcionarles a las plantas condiciones más favorables para el crecimiento, tanto climático como nutricional. Esto permite acondicionar las plántulas y prepararlas para establecerlas en sitios con variadas restricciones provenientes principalmente de limitaciones edafoclimáticas. Razón por la cual, los programas de restauración ecológica y/o reforestación consideran indispensable mejorar la fertilización y su esquema de aplicación, a fin de satisfacer la demanda local, dada la utilidad de esta especie para la conservación de las zonas restauradas y sus usos (Ministerio de Agricultura Ganadería, Acuacultura y Pesca / Cooperación Técnica Alemana, 2017)

Diversas estrategias de fertilización pueden ser utilizadas para inducir ciertas características morfológicas y fisiológicas en las plantas, de modo que estas respondan haciéndose más resistentes o aumentando su potencial de crecimiento (Quiroz, García, González, Chung \& Soto, 2009, p. 75).

Al momento en que la planta inicia el período de máximo crecimiento vegetativo, demanda altos consumos de nutrientes, los que se debe suministrar mediante fertilización mineral; para estos efectos es recomendable utilizar una formulación de fertilizantes, con mayor concentración de nitrógeno en relación a fósforo y potasio, lo cual favorecerá el crecimiento en altura.

Una forma eficiente de aplicar un programa de fertilización inicial se basa en un análisis nutricional del sustrato. Sobre la base de las deficiencias que en él puedan determinarse se deben incorporar nutrientes específicos que promuevan el crecimiento inicial de las plantas (Escobar, 2007).

Los programas de fertilización, se proyectan con base en los tres macronutrientes principales (NPK); los niveles de fertilización deben ajustarse a cada una de las tres etapas de desarrollo de la plántula en vivero (Trujillo, 2015).

El estudio de Rosero (2014), evalua dos fertilizantes de lenta liberación en la propagación asexual de Polylepis racemosa producida en suelo negro de páramo y envases de polietileno. El mejor efecto con fertilización se obtuvo con el fertilizante químico granular formado: NPK, Mg $(16+8+12+2 \mathrm{Mg})$ y microelementos, en una dosis 4 g planta $^{-1}$, cuya influencia se determinó con la cuantificación de mayor número de brotes, crecimiento 
en longitud del brote y en el volumen del sistema radicular en la propagación mediante esquejes (p. 29).

En este contexto, los requerimientos de la especie varían en cada una de las etapas, tanto en calidad como en su equilibrio, debido a que cada especie posee diferentes reservas con base en su tipo de estructura de propagación, la cual es una característica de compatibilidad de las fuentes nutritivas a utilizar cuando se combinan entre sí para su aplicación. Aunque todas las fases del manejo del vivero son importantes, el sustrato y las prácticas que tienen que ver con él, constituyen el centro de todo programa de manejo de la fertilización (Gómez, 2006).

Para lograr plantas con mejores características morfológicas, además de las fisiológicas, es necesario el desarrollo de técnicas culturales desde el vivero. El tipo de sustrato, el contenedor a utilizar, la calidad de propágulos, el régimen de nutrición y el manejo adecuado del riego, son los elementos principales a considerar para obtener plántulas de calidad a un precio razonable (Leyva et al., 2008).

Factores importantes que contribuyen al éxito de un programa nutricional son: la calidad del agua de riego, la frecuencia e intensidad del riego, la calidad del sustrato, las condiciones meteorológicas, el control oportuno de patógenos (Duryea, 1985). Por tanto, en este estudio se planteó evaluar el efecto de la aplicación mediante fertirriego con NPK en la etapa de crecimiento del Polylepis racemosa.

\section{Materiales y métodos}

\section{1. Área de estudio}

La presente investigación se realizó en el vivero forestal de la comunidad TiocajasPalacio, ubicada al Sur - oeste del Cantón Guamote, Provincia de Chimborazo, a $13 \mathrm{~km}$ de la cabecera cantonal. Geográficamente el vivero se localiza a $2^{\circ} 5^{\prime} S$ y $78^{\circ} 45^{\prime} \mathrm{W}$, a una altitud de $3660 \mathrm{msnm}$. La precipitación media anual es $1200 \mathrm{~mm}$; la temperatura media es de $11^{\circ} \mathrm{C}$, con una humedad relativa $80 \%$ y una velocidad del viento de $6 \mathrm{~m} \mathrm{~s}^{-1}$ (Serie 20122015 de la Estación Meteorológica Totorillas, 2015). Se categoriza dentro de la formación: Bosque Húmedo Pre-montano (Ministerio del Ambiente del Ecuador, 2012).

\subsection{Abastecimiento de plántulas}

El material vegetal utilizado para la evaluación del ensayo correspondió a plántulas de cuatro meses de edad producidas en fundas de 10,16 x 15,24 cm a partir de estacas de $20 \mathrm{~cm}$ procedentes de plantas madres existentes en el vivero de la comunidad Chauzán Totorillas, parroquia Palmira, cantón Guamote. La plantación se realizó introduciendo 6 $\mathrm{cm}$ en el sustrato, dejando una yema en contacto. Se seleccionó aquellas plántulas que presentaron una altura de 9 a $14 \mathrm{~cm}$ y mayor vigor, el día 5 de julio del 2017. (González et al., 2009). Las plántulas seleccionadas fueron llevadas al vivero de la comunidad Tiocajas-Palacio.

\subsection{Establecimiento de la investigación en campo}

Las plántulas adquiridas se ubicaron dentro de un umbráculo provisto de malla plástica (malla Raschel) en la parte superior con una superficie 10 metros de longitud por $7 \mathrm{~m}$ de ancho. El área fue dividida en 28 parcelas de $75 \times 75 \mathrm{~cm}$, donde se realizó el trasplante a fundas de $8 \times 10$ pulgadas. La actividad se realizó manualmente, para asegurar una buena distribución y compactación, así como la rigidez deseada del envase. El sustrato utilizado es una mezcla de $20 \%$ de tierra negra $+20 \%$ de cascarilla de arroz + $10 \%$ abono orgánico $+50 \%$ de tierra agrícola del sitio. Las características químicas del sustrato al inicio del experimento, se determinaron en el Laboratorio de Suelos de la Facultad de Recursos Naturales (ESPOCH, 2017). Los resultados obtenidos fueron: 7 \% 
de materia orgánica que corresponde a un nivel alto. El potencial de hidrógeno $\mathrm{pH}$ es de 6.0 interpretado ligeramente ácido; los contenidos nutricionales de $13,4 \mathrm{mg} . \mathrm{L}^{-1}$; $11,9 \mathrm{mg} \cdot \mathrm{L}^{-1} ; 108,5 \mathrm{mg} \cdot \mathrm{L}^{-1}$ se enmarcan en niveles bajos para nitrógeno, fósforo y potasio respectivamente.

\subsection{Características de las fuentes para preparar las soluciones}

En la Tabla 1 se muestran la composición química y la concentración de los elementos que forman parte de las fuentes utilizadas en la elaboración de las soluciones.

Tabla 1. Composición química de los fertilizantes

\begin{tabular}{|l|c|l|}
\hline Fuente & Fórmula química & Concentración (\%) \\
\hline Nitrato de amonio & $\left(\mathrm{NH}_{4} \mathrm{NO}_{3}\right)$ & $33-34 \mathrm{~N}$ \\
\hline Nitrato de potasio & $\left(\mathrm{KNO}_{3}\right)$ & $13-14 \mathrm{~N}, 44-46 \mathrm{~K}_{2} \mathrm{O}$ \\
\hline Fosfato mono potásico & $\left(\mathrm{KH}_{2} \mathrm{PO}_{4}\right)$ & $51 \mathrm{P}_{2} \mathrm{O}_{5}, 35 \mathrm{~K}_{2} \mathrm{O}$ \\
\hline
\end{tabular}

Fuente: Laboratorio marasas (2016).

La aplicación de las dosis de nitrógeno, fósforo y potasio tuvo una duración de 16 semanas, desde julio a octubre de 2017 , lo cual abarcó la fase de pleno crecimiento.

\subsection{Preparación de soluciones nutritivas}

El cálculo del volumen de riego, se realizó de acuerdo a la metodología de infiltración (Pizarro \& Flores, 2008), en el laboratorio de hidráulica de la ESPOCH (2017), donde se determinó que 3,5 L.m $\mathrm{m}^{2-1}$.día-1 es la cantidad que se debe aplicar al sustrato.

El nivel deseado de nutrientes para las plantas, se determinó con base al análisis nutricional del sustrato, la etapa de crecimiento de la especie, la concentración óptima de fertilizantes líquidos de acuerdo a Landis et al., (1989), así como la compatibilidad de los fuentes nutritivas elegidas cuando se combinan entre sí (Villalobos et al., 2016). Para el cálculo del régimen de nutrientes se utilizó la Ecuación 1.

$$
\mathbf{C}=\frac{\mathrm{MxF \times L \times FC}}{\mathrm{FN}}
$$

\section{Donde:}

$\mathrm{C}=$ cantidad de producto a utilizar

$\mathrm{M}=$ concentración del producto

$\mathrm{F}=$ factor de dilución

$\mathrm{L}=$ cantidad de agua

$\mathrm{Fc}=$ constante

$\mathrm{FN}=$ factor

\subsection{Diseño experimental}

Se estableció un Diseño de Bloques Completamente al Azar (DBCA), con un arreglo factorial con seis tratamientos más un testigo. Cada tratamiento consistió de cuatro repeticiones, con veinte plantas como unidades experimentales (80 plantas por tratamiento). Los tratamientos utilizados se describen a continuación.

Las dosis de fuentes nutritivas consideradas se diluyeron en agua, hasta alcanzar los 44,8 L. Para fertilizar se utilizó el método de Drench (Boix, 2012, p. 60). Se consideró el esquema de fertirriego programado ( Tabla 2), el cual se aplicó entre dos y tres veces a la semana respectivamente (Escobar, 2007). 
Tabla 2. Distribución de los tratamientos en $\mathrm{mg} \cdot \mathrm{L}^{-1}$ de NPK

\begin{tabular}{|c|c|c|c|}
\hline № Trat & Código & $\begin{array}{l}\text { Concentración } \\
\left(\mathrm{mg}^{-1} \mathrm{~L}^{-1}\right) \text { de NPK }\end{array}$ & Descripción: cantidad de fertilizantes en gramos \\
\hline T1 & $\mathrm{D} 1 \mathrm{~F} 1$ & $100-50-100$ & $\begin{array}{l}{\left[4,61 \mathrm{~g}\left(\mathrm{KNO}_{3}\right)+9,91 \mathrm{~g}\left(\mathrm{KH}_{2} \mathrm{PO}_{4}\right)+11,47 \mathrm{~g}\left(\mathrm{NH}_{4} \mathrm{NO}_{3}\right)\right] / 44,8} \\
\mathrm{~L} / 2 \text { veces/semana }\end{array}$ \\
\hline T2 & D1F2 & $100-50-100$ & $\begin{array}{l}{\left[4,61 \mathrm{~g}\left(\mathrm{KNO}_{3}\right)+9,91 \mathrm{~g}\left(\mathrm{KH}_{2} \mathrm{PO}_{4}\right)+11,47 \mathrm{~g}\left(\mathrm{NH}_{4} \mathrm{NO}_{3}\right)\right] / 44,8} \\
\mathrm{~L} / 3 \text { veces/semana }\end{array}$ \\
\hline T3 & $\mathrm{D} 2 \mathrm{~F} 1$ & $150-75-150$ & $\begin{array}{l}{\left[6,91 \mathrm{~g}\left(\mathrm{KNO}_{3}\right)+14,86 \mathrm{~g}\left(\mathrm{KH}_{2} \mathrm{PO}_{4}\right)+17,2 \mathrm{~g}\left(\mathrm{NH}_{4} \mathrm{NO}_{3}\right)\right] / 44,8} \\
\mathrm{~L} / 2 \text { veces/semana }\end{array}$ \\
\hline T4 & D2F2 & $150-75-150$ & $\begin{array}{l}{\left[6,91 \mathrm{~g}\left(\mathrm{KNO}_{3}\right)+14,86 \mathrm{~g}\left(\mathrm{KH}_{2} \mathrm{PO}_{4}\right)+17,2 \mathrm{~g}\left(\mathrm{NH}_{4} \mathrm{NO}_{3}\right)\right] / 44,8} \\
\mathrm{~L} / 3 \text { veces/semana }\end{array}$ \\
\hline T5 & D3F1 & $200-100-200$ & $\begin{array}{l}{\left[9,22 \mathrm{~g}\left(\mathrm{KNO}_{3}\right)+19,82 \mathrm{~g}\left(\mathrm{KH}_{2} \mathrm{PO}_{4}\right)+22,94 \mathrm{~g}\left(\mathrm{H}_{4} \mathrm{NO}_{3}\right)\right] / 44,8} \\
\mathrm{~L} / 2 \text { veces/semana }\end{array}$ \\
\hline T6 & D3F2 & $200-100-200$ & $\begin{array}{l}{\left[9,22 \mathrm{~g}\left(\mathrm{KNO}_{3}\right)+19,82 \mathrm{~g}\left(\mathrm{KH}_{2} \mathrm{PO}_{4}\right)+22,94 \mathrm{~g}\left(\mathrm{H}_{4} \mathrm{NO}_{3}\right)\right] / 44,8} \\
\mathrm{~L} / 3 \text { veces/semana }\end{array}$ \\
\hline T7 & Testigo & Sin solución & \\
\hline
\end{tabular}

Dosis baja: 100-50-100 mg.L-1 NPK (D1); Dosis media: 150- 75- 150 mg.L-1 NPK (D2); Dosis alta: 200-100200 mg.L-1 NPK (D3). NPK: Nitrógeno, fósforo y potasio. Frecuencia de aplicación: 2 veces semana ${ }^{-1}(\mathrm{~F} 1), 3$ veces semana-1 $^{-1}$ F2).

\subsection{Labores culturales}

En base a la observación de síntomas causados por insectos de la familia Cucurlionidae, defoliadores de hojas, se aplicó un control químico con una frecuencia de 15 días durante el período de investigación; se realizó el control químico mediante cipermetrina en una dosis de $1 \mathrm{~cm}^{3} \cdot \mathrm{L}^{-1}$ conjuntamente con la actividad de deshierba (Boix, op.cit, p., 127). Se cuidó la compatibilidad de los fertilizantes cuando se combinan entre sí e inclusive con la composición química del agua de riego (Gómez, 2006).

\subsection{Evaluación en el área de crecimiento}

Durante la fase de crecimiento, fueron seleccionadas 5 plantas, ubicadas en la parte central de cada parcela neta, para eliminar el efecto de borde; las plantas fueron cuantificadas en altura total (hT) y número de hojas $\left(\mathrm{N}^{\circ} \mathrm{H}\right)$, a los 30, 60, 90 y 120 días después del trasplante.

\subsection{Análisis estadístico.}

Se probó el comportamiento normal de las variables, altura total (hT) y número de hojas $\left(\mathrm{N}^{\circ} \mathrm{H}\right)$ mediante el estadístico Shapiro-Wilk; al verificar que no se cumple el supuesto de normalidad en las dos variables, se aplicó la prueba de Kruskall Wallis en el programa INFOSTAT (Balzarini et al., 2008).

\section{Resultados}

\subsection{Altura total de la planta}

El mayor crecimiento en altura total de planta lo reportó la aplicación de 200-100$200 \mathrm{mg} . \mathrm{L}^{-1}$ de NPK, tres veces por semana (T6) con valores promedio de 15,20; 21,14 y $28,16 \mathrm{~cm}$ a los 60,90 y 120 días respectivamente. Las menores alturas de planta, se lograron con la aportación de 100-50-100 mg. L-1 de NPK, dos veces por semana (T1) con índices de 12,66; 15,28 y 17,95 cm durante los periodos evaluados. Las diferencias de crecimiento entre los siete tratamientos evaluados obtuvieron un valor $p<0.0001$ (Tabla 3). 
Tabla 3. Prueba de kruskal Wallis de la variable altura de planta a los 30, 60, 90 y 120 días en la fase de crecimiento rápido $(\mathrm{cm})$

\begin{tabular}{|c|c|c|c|c|c|c|c|}
\hline $\begin{array}{l}\text { Variable } \\
\text { medida }\end{array}$ & Tratamientos & $\mathbf{N}$ & Medias & D.E. & Medianas & H & p \\
\hline \multirow{7}{*}{ Altura 30 días } & 1 & 20 & 10,83 & 1,17 & 11,00 & 7,44 & 0,2729 \\
\hline & 2 & 20 & 11,16 & 1,68 & 11,00 & & \\
\hline & 3 & 20 & 10,76 & 1,29 & 11,00 & & \\
\hline & 4 & 20 & 11,39 & 1,71 & 11,00 & & \\
\hline & 5 & 20 & 10,81 & 1,28 & 10,4 & & \\
\hline & 6 & 20 & 11,02 & 1,71 & 10,4 & & \\
\hline & 7 & 20 & 11,74 & 1,22 & 11,4 & & \\
\hline \multirow{7}{*}{ Altura 60 días } & 1 & 20 & 12,66 & 1,12 & 13,00 & 32,01 & $<0,0001$ \\
\hline & 2 & 20 & 13,66 & 1,94 & 13,00 & & \\
\hline & 3 & 20 & 13,93 & 1,04 & 14,00 & & \\
\hline & 4 & 20 & 14,61 & 2,11 & 13,95 & & \\
\hline & 5 & 20 & 14,54 & 1,36 & 14,15 & & \\
\hline & 6 & 20 & 15,20 & 1,77 & 14,30 & & \\
\hline & 7 & 20 & 13,77 & 1,31 & 14,00 & & \\
\hline \multirow{7}{*}{ Altura 90 días } & 1 & 20 & 15,28 & 1,23 & 15,00 & 69,11 & $<0,0001$ \\
\hline & 2 & 20 & 16,86 & 2,36 & 16,65 & & \\
\hline & 3 & 20 & 17,05 & 1,61 & 17,25 & & \\
\hline & 4 & 20 & 18,31 & 2,52 & 18,00 & & \\
\hline & 5 & 20 & 18,81 & 1,71 & 18,30 & & \\
\hline & 6 & 20 & 21,14 & 2,20 & 21,30 & & \\
\hline & 7 & 20 & 16,02 & 1,36 & 16,15 & & \\
\hline \multirow{7}{*}{ Altura 120 días } & 1 & 20 & 17,95 & 1,73 & 18 & 92,97 & $<0,0001$ \\
\hline & 2 & 20 & 19,92 & 2,93 & 19,95 & & \\
\hline & 3 & 20 & 20,67 & 2,39 & 21,25 & & \\
\hline & 4 & 20 & 22,97 & 2,93 & 23,20 & & \\
\hline & 5 & 20 & 24,51 & 2,26 & 24,15 & & \\
\hline & 6 & 20 & 28,16 & 2,25 & 28,15 & & \\
\hline & 7 & 20 & 18,04 & 1,45 & 18,10 & & \\
\hline
\end{tabular}

Número de plantas muestreadas por tratamiento (N), Desvío estándar (D.E), Estadístico de prueba de Kruskal Wallis $(\mathrm{H})$, El valor $p$ asociado $(p)$.

\subsection{Número de hojas}

La prueba de Kruskal Wallis indica que la variable número de hojas a los 60, 90 y 120 días después del trasplante, presenta diferencias estadísticas $(p<0.0003 ; p<0.0001)$ (Tabla 4).

La mayor cantidad de hojas a los 60 y 90 días, se registró al aplicar 200-100-200 mg. $\mathrm{L}^{-1}$ de NPK, dos veces por semana (T5) con valores promedios de 10,10 y 17,40 hojas.

El menor número de hojas por planta mostró el aporte de 100-50-100 mg.L-1 de NPK, dos veces por semana (T1) con índices de 7,60 y 13,30 hojas.

A los 120 días, con el aporte de 200-100-200 mg. L-1 de NPK, tres veces por semana (T6), se obtiene el mayor número de hojas con 28,95 y en menor cantidad, se reportó un comportamiento similar en los tratamientos uno y siete con 19,15 hojas respectivamente. 
Tabla 4. Prueba de kruskal Wallis de la variable número de hojas a los 30, 60, 90 y 120 días durante la etapa de crecimiento rápido

\begin{tabular}{|c|c|c|c|c|c|c|c|}
\hline $\begin{array}{l}\text { Variable } \\
\text { medida }\end{array}$ & Tratamientos & $\mathbf{N}$ & Medias & D.E. & Medianas & H & $\mathbf{p}$ \\
\hline \multirow{7}{*}{$\begin{array}{c}\text { Número de } \\
\text { hojas } 30 \text { días }\end{array}$} & 1 & 20 & 4,20 & 1,28 & 4,00 & 6,82 & 0,3139 \\
\hline & 2 & 20 & 4,55 & 1,76 & 4,00 & & \\
\hline & 3 & 20 & 4,95 & 2,42 & 4,00 & & \\
\hline & 4 & 20 & 4,85 & 2,08 & 4,50 & & \\
\hline & 5 & 20 & 5,35 & 1,39 & 5,00 & & \\
\hline & 6 & 20 & 4,60 & 1,43 & 4,5 & & \\
\hline & 7 & 20 & 5,1 & 1,52 & 5,00 & & \\
\hline \multirow{7}{*}{$\begin{array}{l}\text { Número de } \\
\text { hojas } 60 \text { días }\end{array}$} & 1 & 20 & 7,60 & 1,27 & 8,00 & 24,72 & $<0,0003$ \\
\hline & 2 & 20 & 8,15 & 2,28 & 7,50 & & \\
\hline & 3 & 20 & 8,80 & 2,38 & 8,00 & & \\
\hline & 4 & 20 & 9,25 & 2,22 & 9,00 & & \\
\hline & 5 & 20 & 10,10 & 1,65 & 10,00 & & \\
\hline & 6 & 20 & 9,95 & 2,19 & 9,50 & & \\
\hline & 7 & 20 & 9,00 & 1,49 & 9,00 & & \\
\hline \multirow{7}{*}{$\begin{array}{l}\text { Número de } \\
\text { hojas } 90 \text { días }\end{array}$} & 1 & 20 & 13,30 & 2,05 & 13,00 & 44,13 & $<0,0001$ \\
\hline & 2 & 20 & 14,10 & 2,55 & 13,50 & & \\
\hline & 3 & 20 & 15,00 & 3,03 & 15,00 & & \\
\hline & 4 & 20 & 15,75 & 2,29 & 15,50 & & \\
\hline & 5 & 20 & 17,40 & 2,44 & 17,50 & & \\
\hline & 6 & 20 & 17,10 & 2,07 & 17,00 & & \\
\hline & 7 & 20 & 13,60 & 1,76 & 13,50 & & \\
\hline \multirow{7}{*}{$\begin{array}{c}\text { Número de } \\
\text { hojas } 120 \text { días }\end{array}$} & 1 & 20 & 19,15 & 1,73 & 19,00 & 88,95 & $<0,0001$ \\
\hline & 2 & 20 & 21,80 & 2,65 & 22,00 & & \\
\hline & 3 & 20 & 23,40 & 3,39 & 23,00 & & \\
\hline & 4 & 20 & 24,20 & 2,84 & 24,00 & & \\
\hline & 5 & 20 & 26,95 & 2,98 & 27,50 & & \\
\hline & 6 & 20 & 28,95 & 2,65 & 29,00 & & \\
\hline & 7 & 20 & 19,15 & 2,16 & 19,00 & & \\
\hline
\end{tabular}

Número de plantas muestreadas por tratamiento (N), Desvío estándar (D.E), Estadístico de prueba de Kruskal Wallis $(H)$, El valor $p$ asociado $(p)$.

\section{Discusión}

A los 30 días no se determinó un crecimiento significativo en altura de planta entre los tratamientos, las respuestas en crecimiento producto del fertirriego no son inmediatas (Materán et al. 2004). Debido a que se encuentra en una etapa de transición entre la fase de establecimiento y la de crecimiento a nivel de vivero (Schinelli \& Martínez, 2010), la fertilización aplicada mediante fertirriego influye directamente en el crecimiento de las plantas en vivero. Mostrando el efecto de los macronutrientes en procesos fisiológicos, cuya manifestación más evidente es el crecimiento que va en aumento. Llegó a obtenerse como el mejor promedio en altura total de 15,20; 21,14 y $28,16 \mathrm{~cm}$ con la aplicación de 200-100-200 mg. L-1 NPK, tres veces por semana (T6) dosis alta, para los 60, 90 y 120 días respectivamente. 
Etapa de crecimiento resultado de la aplicación de un nivel elevado de $\mathrm{N}$ que promueve la división y elongación celular, el $\mathrm{P}$, estimula principalmente el desarrollo del sistema radicular y participa en la generación de la energía (ATP) que requiere la planta para su crecimiento y desarrollo. En cuanto al potasio, aumenta su vigor y resistencia a las heladas (Trujillo, 2010, p. 39). Durante esta fase se pretende lograr que las plantas alcancen una mayor altura acorde a su etapa de crecimiento y un desarrollo radicular fortalecido.

La variable altura total de planta de la especie en estudio resultó $8,18 \%$ menor al valor de 30,67 cm reportado en el cantón Olmedo-Cayambe con una precipitación 795,7 $\mathrm{mm}$, una temperatura de $11,6^{\circ} \mathrm{C}$, con una humedad relativa del $83 \%$, a una altitud de 3103 msnm, utilizando el sustrato formado por: $33,33 \%$ de tierra negra $+50 \%$ tierra agrícola $+16,67 \%$ arena y 6 meses de edad (Gualavisi, 2008, p. 21). Resultado que se ubica entre 25 a $35 \mathrm{~cm}$ de altura, parámetro que indica una calidad morfológica adecuada de manera general para latifoliadas (Sáenz et al., 2010).

Los resultados obtenidos para la variable número de hojas mostraron que todas las plantas sometidas a los diversos niveles y frecuencias de aplicación nutrimentales incrementaron en el número de hojas (Tabla 4). Se determinó que las plantas fertilizadas con 200-100-200 mg. $\mathrm{L}^{-1} \mathrm{NPK}$, dos veces por semana (T5), desarrollaron en promedio la mayor cantidad de hojas a los 60 y 90 días, destacándose para los 120 días la aplicación de 200-100-200 mg. L $^{-1} \mathrm{NPK}$, tres veces por semana (T6), dosis alta, en contraste con las plantas no fertilizadas que corresponden al testigo.

Las plantas que se fertirrigaron con el tratamiento 6 , superaron en $33,85 \%$ la cantidad de hojas, comparadas con las plantas testigo.

De los 60 a 120 días se obtuvo la mayor cantidad de hojas; mejor crecimiento en altura de las plantas sometidas al tratamiento 6 , debido a la respuesta asociada a la actividad fotosintética más eficiente, la cual garantiza mayor captura de radiación solar incidente por su área foliar (Lok \& Suárez, 2014).En este proceso se producen cantidades superiores de foto sintetizados y la planta produce más alimento y energía, brindando vigorosidad a las mismas (Trujillo, op.cit, p., 39).

\section{Conclusiones}

Al fertilizar con la dosis alta que comprende la aplicación de 200-100-200 mg..-1 de NPK tres veces por semana a los 120 días, se obtuvo la mejor altura de planta y el mayor número de hojas logrando un buen crecimiento en las plantas de Polylepis racemosa. En contraste con el menor crecimiento en altura de planta, que mostró el uso de 100-50-100 mg. $\mathrm{L}^{-1}$ de NPK (T1) y el menor número de hojas, se reportó en los tratamientos uno y siete. Los resultados indican que el yagual responde de manera positiva al uso de la solución nutritiva en dosis alta y con una frecuencia de aplicación de tres veces por semana en su primera fase de crecimiento a nivel de vivero.

Se sugiere evaluar sus atributos morfológicos, fisiológicos (calidad de planta) en la fase de endurecimiento y considerar las actividades complementarias de manejo a nivel de vivero con el propósito de lograr plantas más equilibradas para su mejor funcionamiento en el sitio definitivo en términos de supervivencia.

\section{Bibliografía}

Añazco, M. (2008). "Usos medioambientales de las plantas". In: de la Torre, L., H. Navarrete, P. Muriel M., M. J. Macía \& H. Balslev (eds.). Enciclopedia de las Plantas Útiles del Ecuador. Herbario QCA de la Escuela de Ciencias Biológicas de la Pontificia Universidad Católica del Ecuador \& Herbario AAU del Departamento de Ciencias Biológicas de la Universidad de Aarhus. Quito, EC \& Aarhus. Consultado el 20 de noviembre de 2017. Disponible en http://www.biologia.puce.edu.ec /imagesFTP/10457.Medioambiental.pdf 
Balzarini, M. G.; Gonzalez, L.; Tablada, M., et. al. (2008). Infostat. Manual del Usuario. Córdova, Argentina: Brujas.

Boix, E. (2012). Operaciones básicas de producción y mantenimiento de plantas en viveros y centros de jardinería. España: Ediciones Paraninfo.

Duryea, M.L (Ed.). (1985). Proceedings: Evaluating seedling quality: principles, procedures and predictive abilities of major tests. Workshop: Forest Research Laboratory Oregon State University.

Escobar, R. (2007). Manual de viverización. Eucalyptus globulus a raíz cubierta. Concepción; Chile: Proyecto Innova Chile - INFOR. Instituto Forestal.

Estación Meteorológica Totorillas (2016). Datos climáticos de la serie 2013-2015. Riobamba.

Gómez, M. (2006). Manual técnico de fertilización de cultivos. Santafé de Bogotá: Editorial produmedios.

González, M., Quiroz, I., García, E., y Soto, H. (2009). "Plantas de Nothofagus glauca (Phil.) Krasser. Ensayos de Germinación y Producción”. En: Revista Chile Forestal, 344,pp. 40-44.

Gualavisi, L. (2008). Comportamiento de Polylepis racemosa en vivero mediante propagación vegetativa utilizando cuatro longitudes de estacas en platabandas a nivel en tres diferentes pisos altitudinales Cayambe 2008. Tesis de pregrado, Facultad de Ciencias Agropecuarias y Ambientales, Universida Politécnica Salesiana Sede Quito, Ecuador.

Laboratorio de suelos de la Facultad de Recursos Naturales de la ESPOCH. (2017). Resultados del Análisis de sustrato del ensayo Polylepis racemosa a nivel de vivero. Riobamba. Ecuador.

Laboratorio de hidráulica de la ESPOCH. (2017). Determinación de volumen de riego en el sustrato del ensayo Polylepis racemosa. Riobamba. Ecuador.

Laboratoriomarasas (2016)."Fertilizantes". Consultado el 10 de marzo de 2018. Disponible en http://www.laboratoriomarasas.com.ar/sites/www.laboratoriomarasas.com.ar/files /COMPOSICI\%C3\%93N\%20QU\%C3\%8DMICA\%20DE\%20FERTILIZANTES_0.pdf

Landis, T.D.; R.W. Tinus; S.E. Mc Donald y J.P. Barnett. (1989). Seedling Nutrition and Irrigation. Vol. 4. The Container Tree Nursery Manual. Agric. Handbook No 674. USDA.

Leyva, R. F.; Rosell P. R.; Ramírez R. A., y Romero R. I. (2008). Manejo de endurecimiento por riego para elevar la calidad de las plantas de Eucalyptus sp. cultivadas en vivero de la Unidad Silvícola Campechuela. Universidad de Granma. Central del Batey. Campechuela. Granma. Cuba.

Lok, S., y Suárez, Y. (2014). "Efecto de la aplicación de fertilizantes en la producción de biomasa de Moringa oleifera y en algunos indicadores de suelo durante el enraizamiento". En: Revista Cubana de Ciencia Agícola, 4, pp. 398-403.

Materán M, R.; Coopman, D.; Ríos, M., y Sánchez. (2004). Efecto del estrés nutricional de $\mathrm{N}$ sobre el crecimiento de plantas de Eucalyptus globulus Labill. Segundo Congreso Chileno de Ciencias Forestales. Valdivia. Chile.

Ministerio del Ambiente del Ecuador. (2012). Sistema de clasificación de los ecosistemas del Ecuador continental. Quito: Subsecretaria de Patrimonio Natural.

Ministerio de Agricultura Ganadería, Acuacultura y Pesca / Cooperación Técnica Alemana. (2017). Buenas Prácticas Agrarias para enfrentar al Cambio Climático en Ecuador. Consultado el 28 de diciembre de 2017. Disponible en http://balcon.magap.gob.ec /mag01/magapaldia/libro/BPA\%20MAGAP.pdf

Pizarro, R., y Flores, J. (2008). "Módulo 3 Curvas de infiltración”. En: Sociedad Estándares de Ingeniería para Aguas y Suelos. Consultado el 20 de noviembre de 2017.Disponible en http://eias.utalca.cl/Docs/pdf/Publicaciones/manuales/c_modulo curva_infiltracion.pdf

Quiroz, I.; Gārcía, E.; González, M., et. al. (2009). Vivero Forestal: Producción de plantas nativas a raíz cubierta. Chile: Centro tecnológico de la planta forestal. 
Rosero, J. (2014). Evaluación de dos fertilizantes de lenta liberación en la propagación asexual de yagual (Polylepis racemosa) en la comunidad San José del Guanto, cantón Quero, Tungurahua. Tesis de pregrado, Facultad de Ciencias Agropecuarias, Universidad Técnica de Ambato, Ecuador.

Sáenz, R. J. T.; Villaseñor R. F. J.; Muñoz F. H. J., et. al. (2010). Calidad de planta en viveros forestales de clima templado en Michoacán. Folleto Técnico Núm. 17. SAGARPA-INIFAP-CIRPAC-Campo Experimental Uruapan. Uruapan, Michoacán, México. 48 p.

Schinelli, T., y Martínez, A. (2010). Viverización de Especies Nativas de Nuestra Región. Parte 2: Viverización en Condiciones Controladas. Presencia N 55: 26-30.

Tisdale, S.L.; W.L. Nelson; J. Beaton y J. Havlin. (1993). Soil Fertility and Fertilizers (5 ${ }^{\text {ta }}$ ed.), New York: Macmillan Pub.

Trujillo, E. (2010). Manual de Árboles. Investigaciones Forestales. Colombia: El Semillero.

Trujillo, E. (2015). Guia de reforestación: Ilustrada, aumentada y corregida. Colombia: EI Semillero.

Villalobos, S., Fernández, R., Godoy, H., \& Rodríguez, A. (2016). Preparacion de soluciones nutritivas para cultivos hortícolas en hidroponia. Tlaxcala: Grupo Gráfico Salinas.

Villarpando, D.; Villarpando, P.,y Villalobos, J.(2008). "Fichas botánicas de especies agroforestales nativas y naturalizadas aptas para tierrasaltoandinas". Proyecto de Adaptación al Impacto del Retroceso Acelerado de los Glaciares en los Andes Tropicales (PRAA) Consultado el 20 de febrero de 2018. Disponible en http://www.ecosaf.org/altiplano/Fichas\%20botanicas\%20CARE.pdf 